\title{
Extending Mobile Interfaces with External Screens
}

\author{
Julian Seifert $^{1}$, Dennis Schneider ${ }^{2}$, and Enrico Rukzio ${ }^{1}$ \\ ${ }^{1}$ Ulm University, Ulm, Germany \\ \{julian.seifert, enrico.rukzio\}@uni-ulm.de \\ ${ }^{2}$ University of Duisburg-Essen, Essen, Germany \\ dennis.schneider_1@uni-due.de
}

\begin{abstract}
Mobile phones allow for the use of all kinds of applications, and their mobile applications often provide similar functionalities as desktop applications. However, they are constrained by the limited screen size of the mobile device. Accordingly, designs of mobile user interfaces require optimization for small screens. As a consequence, users are provided with less context and often have to switch views or resize content such as maps or pictures. We present MobIEs ${ }^{1}$, a novel approach for extending mobile user interfaces by using external screens (e.g., the mobile phone and a large screen). Users can utilize more space and can thus overview a larger information context. We present a novel interaction and application concept and describe how user interfaces can be spanned across displays. Further, we contribute an original approach for using Near Field Communication to detect the devices' spatial relation. We report on a user study which compared MobIES with standard mobile settings. Results from the system usability scale show that interaction with MobIES is subjectively more usable. Furthermore, it provides higher perceived information clarity and supports faster sharing of information to others.
\end{abstract}

Keywords: Mobile phones, distributed user interfaces, interaction.

\section{Introduction}

Today's mobile phones enable users to perform a large variety of tasks in mobile contexts. Given the increased computing power, battery capacity, and data connectivity, users can perform the same tasks as by using traditional personal computers (e.g., browsing the web, viewing and editing photos). One of the limiting factors is the screen size of the mobile devices [2]. The screen size affects users mainly in two ways: First, only a limited amount of information can be displayed on the screen at once. Hence users often have to change the view (i.e., zooming in or out, switching between different screens). Second, collaboration with co-located persons is inherently limited, as only a certain amount of people can comfortably view the information.

\footnotetext{
${ }^{1}$ A demo video is available at http: / /youtu. be/dZaCNV64ltk

P. Kotzé et al. (Eds.): INTERACT 2013, Part II, LNCS 8118, pp. 722-729, 2013.

(c) IFIP International Federation for Information Processing 2013
} 


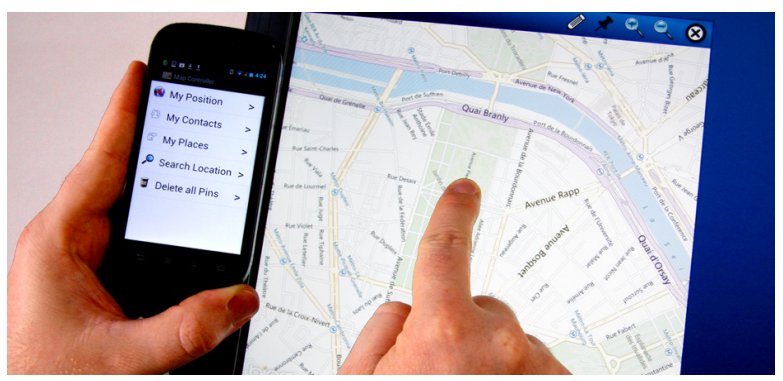

Fig. 1. Spanning a mobile user interface across the mobile phone and an external display, here showing a map application

In this paper, we contribute MobIES, a system that allows users to extend their mobile applications through temporarily spanning the user interface across multiple screens. In short, the technique requires users to touch the border of an available screen (e.g., a public display, TV, or desktop screen) with their phone during the interaction. The system detects this event and initiates the distribution of the user interface across the mobile and the external screen (see Fig. 1). Subsequently, users benefit from the extended screen space which facilitates tasks such as viewing a map, browsing the web, or showing and exchanging pictures to and with other users. When the phone is removed from the border of the external screen, the user interface returns to the original mobile mode. That is, users can take advantage of existing screens in their environments without the need to carry additional hardware.

The contribution of this work is twofold: in this paper, we present the concept and prototype implementation of MobIES and further, we present findings of a user study investigating the system by comparing it with the mobile condition.

\section{Related Work}

Early work on seamlessly connecting devices of different classes investigated how users can share information from their PDAs with others on a large shared device to support collaboration [3]. Integration of personal mobile devices with pre-installed devices in the environment has also been explored [12]. Ullmer et al.'s mediaBlocks showed how data attached to mobile tokens can be transferred to external devices [15]. Hinckley et al. demonstrated how multiple devices with touch screens allow users to drag-and-drop items from one device to another using the stitching technique [6]. Connecting large screens to mobile phones has been investigated [10] while other work focused on creating larger logical screens by combining several devices such as tablet computers [9] and considering the spatial relation of devices and users to each other [8]. Near field communication (NFC) has been used to detect the relative position of mobile devices to larger displays (e.g., [5,11]). Yet no work considered placing NFC tags around an external display which allows a novel way of interaction by using the displays of both devices together. Baur et al. present virtual projection which enables users to transfer data (e.g., pictures) from their phone to a large screen and display it thereon [1]. This approach allows users to take advantage of existing 
displays in their environment. However, the user's interaction is limited to the mobile device. Another approach is to distribute application interfaces on different devices and associated displays [4]. For instance, using mobile devices and large shared displays at which the phone is used as tool by touching the shared display in order to execute actions [13]. Our approach enables users to interact simultaneously with the phone and the extending display. In contrast to the discussed work, MobIES focuses on mobile situations in which the users have the need for more screen space to perform a specific task. The distribution of the user interface of the mobile application onto both devices - both allowing for interaction - increases the user's capabilities.

\section{Concept}

The concept of MobIES is based on users temporarily creating a physical and spatial connection between their mobile device and an external screen to create a larger logical display that consists of the mobile interface and an extended interface on the external screen. We assume that displays in the users' environments can temporarily be used (e.g., public displays, kiosk terminals, TV sets, interactive surfaces, and even screens in cars or airplane seats). User interfaces of mobile applications can display only a limited amount of information (Fig. 2 (left)). By connecting the phone with an external display more screen space is available, thereby allowing for the distribution of the user interface on two screens (Fig. 2 (middle)). Existing work that investigated connecting mobile phones and external screens did not consider the potential of using the mobile and the external screen simultaneously for displaying information. The event of connecting the phone with the display can be sensed, for instance, by using NFC tags that are placed around the external display which is a novel way to use NFC tags for device location detection.
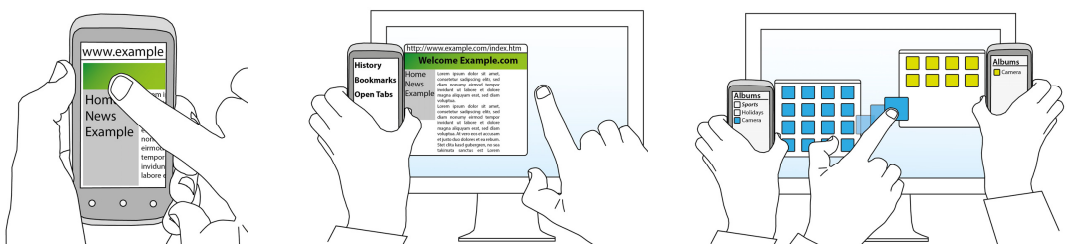

Fig. 2. (left) The mobile user interface allows for the display of a limited amount of information. (middle) Connecting the mobile and an external display extends the available screen space. (right) Items can be shared with others via drag-and-drop to another connected mobile device.

While the phone is connected with an external display, sharing and exchanging data such as pictures, documents, or contact cards can be performed in a straightforward way. Given that the external display supports touch-based interaction, users can simply drag-and-drop items from the external part of their mobile application to the public space. For instance, this can be used in order to leave a message on a bulletin board. In addition, two users can exchange data by both connecting their devices to the same display and drag-and-dropping items from one phone to another (Fig. 2 (right)). 


\section{Implementation}

Our prototype of MobIES consists of two main components. First, a server application running on a PC connected to a host application that is displayed on the stationary touch screen (Dell ST2220T, 22" screen $(1920 \times 1080$ px)). Second, a mobile client (for Android) running on the user's phone (Nexus S; 4" screen $(800 \times 480 \mathrm{px})$ ). The server and the client manage the communication (via TCP over a wireless network) between the distributed application parts. Each application (e.g., a photo album) consists of a mobile component implemented as an Android application and a matching remote part implemented using the Microsoft Surface Toolkit. Depending on which application is active on the mobile phone when the phone touches the rim of the large display, the server launches a matching instance of the remote part of the application in the host application.
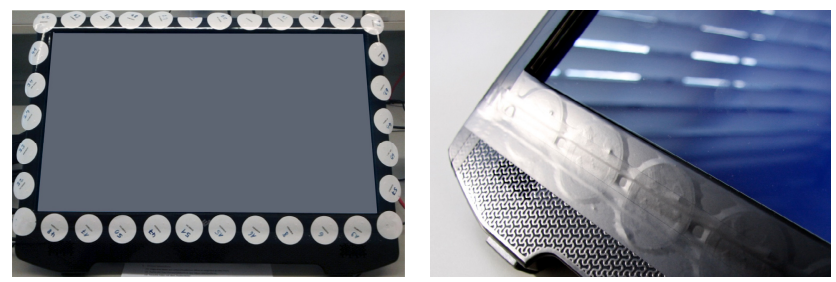

Fig. 3. Display border with NFC tags (left), covered with tape (right)

NFC tags are used to detect when a phone is placed on the border of the large display. NFC is supported by large number of different mobile devices (e.g., Samsung Nexus and Nokia devices). Every 50 millimeters, an NFC tag is placed on the display rim (see Fig. 3). When a phone equipped with an NFC reader is placed on the rim, it reads the tag content. This includes the position on the border, the display server's IP, and the name of the wireless network. If the phone is not connected to the server application, the phone client establishes the connection with the wireless network and connects to the server. Finally, the phone client sends back the tag position and the ID or the currently active mobile application to the server which then launches the remote part of the application.

Using NFC tags allows for the extension of any existing screen to support MobIES interactions. This includes non-touch-enabled displays (e.g., public displays), as users can perform input on the phone while the external display extends the screen space.

\section{$5 \quad$ Evaluation}

We conducted a comparative user study to investigate to what extent MobIES supports users in performing typical mobile tasks. In particular, we were interested in gaining insights concerning usability and how participants perceive this extension of the user interface through holding the phone next to the extending screen compared to the familiar practice of using only mobile phones. 
For the experiment, we implemented based on Schneider et al. [14] three applications that allow users to experience the MobIES concept. These include a photo album, a map, and a web browser application. All applications could be used with an additional external display or as a stand-alone mobile application using only a mobile phone. Using only the mobile phone without the extension of the user interface on an external display was used as a comparative condition for the practical tasks (in the following referred to as the mobile-only or $M O$ option). The features of the applications cover standard functionalities inspired by existing Android applications.
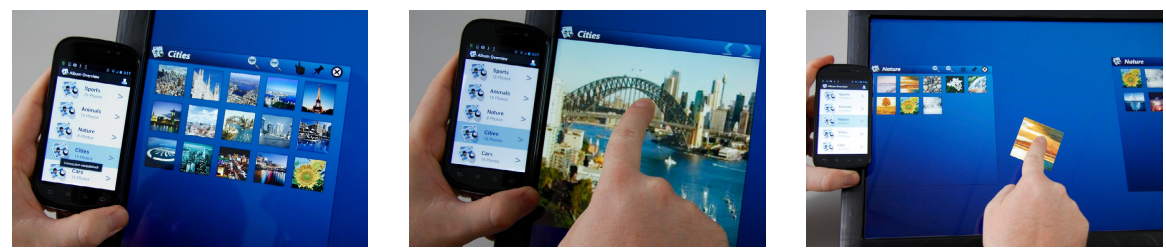

Fig. 4. The photo sharing application: (left) extended overview; (middle) focus on a single image; (right) sharing images with another user by dragging an image from one extended interface to another

In the mobile mode, the photo album application enables users to organize photos taken with the phone in different albums. After selecting an album, contained items are displayed as small thumbnails. Touching a thumbnail activates the full screen mode. When the user launches the extended interface by holding the mobile phone next to the display border, the phone displays the album list and the extended interface shows an overview of picture tiles (Fig. 4 (left)). Which album is displayed can be selected using the list on the mobile interface. Selecting an item in the overview magnifies the picture to fill the application window on the extended interface (Fig. 4 (middle)). For the transfer of pictures from one mobile phone to another, users drag-and-drop items from one extended interface to another (Fig. 4 (right)).

The web browser application provides a history overview and supports tabbed browsing and bookmark management (in both modes). As the user connects the phone to the external display, the phone shows a menu containing options (e.g., History, Open Tabs) and the extended interface shows the corresponding content such as the list of bookmarks (see Fig. 5). For typing in text, the user can use a virtual software keyboard either on the phone or on the external display.

The map application enables users to display addresses of contacts on a map, as well as the selection of points of interests from a list, and searching for places.

Participants were asked to perform a number of tasks via MobIES and the comparative MO option while using a preconfigured mobile phone on which all required data (e.g., pictures or contacts) were available. With the photo album application, participants performed the following tasks: 1) Show the investigator pictures showing people from three different albums; 2) Search for the picture showing the (Eiffel Tower, rocks $\}$ in the albums and delete it. With the map application, participants performed the following tasks: 1) Find the Eiffel Tower / Tower Bridge on the map and show it to the investigator; 2) Show the investigator the addresses of two contacts from the 

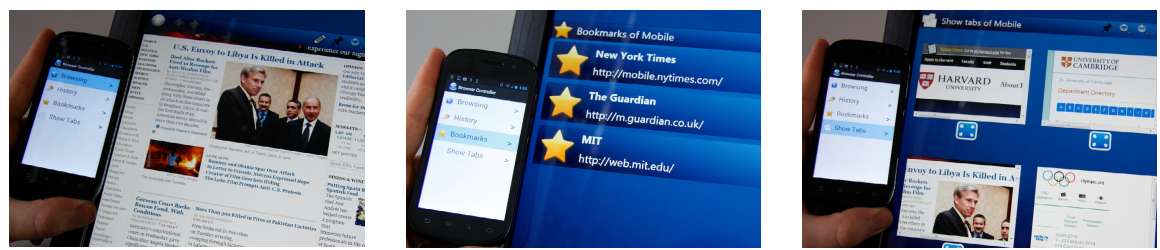

Fig. 5. The web browser application: (left) extended web page view; (middle) selecting bookmarks; (right) browser tab overview

address book as a pin on a map. For the third block of tasks, participants used the browser application: 1) Open the test webpage and look up the contact information of the author; 2) Add a test webpage to the bookmarks and check if the URL was added.

The investigator introduced MobIES and the MO option and participants practiced using them. Then the participants performed a series of tasks, once using MobIES and once as a comparative approach using mobile phones only (MO). The order of systems was counterbalanced and the task order was randomized. Participants filled in a questionnaire regarding usability, including the computer system usability questionnaire [7], after performing the tasks with each system.

We recruited 16 participants (5 females), aged between 20-33 ( $\mathrm{M}=26)$. All participants were students with diverse fields of studies. All participants used smartphones with a touch screen and 14 reported having experience with multi-touch displays. They received 10 Euro after the study session which lasted an average of 45 minutes.

\section{$6 \quad$ Evaluation Results}

On average, each system condition was used for 20 minutes. After each trial, they filled in a questionnaire and rated the system ( $1=$ Strongly disagree; $7=$ Strongly agree). We used the non-parametric Wilcoxon signed-rank test to evaluate differences. Regarding (Q1) "Using the system, I could easily show information to other persons" participants rated the MobIES system significantly higher $(\mathrm{Mdn}=7.0)$ than the MO condition $(\mathrm{Mdn}=3.0)(\mathrm{z}=-3.3, \mathrm{p}=.001)$. Similarly, participants rated MobIES $(\mathrm{Mdn}=7.0)$ significantly higher than $\mathrm{MO}(\mathrm{Mdn}=5.0)$ regarding (Q2) "The system supported sharing of information well" $(\mathrm{z}=-3.3, \mathrm{p}=.001)$. Further, participants rated MobIES higher $(\mathrm{Mdn}=7.0)$ than MO $(\mathrm{Mdn}=3.5)$ in regards to (Q3) "The system supported jointly viewing of information well" $(\mathrm{z}=-3.4, .001)$. Yet both conditions were rated equally concerning (Q4) "Using the system, I often had to change my focus" $(\mathrm{z}=-.4, \mathrm{p}=.72)$. One likely reason is that the larger screen space provided by MobIES spanned across two devices and thus required users to change their focus, much as using only the mobile phone requires switching between different views. Regarding Q5 participants rated MobIES significantly higher $(\mathrm{Mdn}=7.0)$ when compared to MO $(\mathrm{Mdn}=5.0)(\mathrm{z}=-2.9, \mathrm{p}=.004)$.

Participants rated both conditions using the IBM post study system usability questionnaire ( $1=$ Strongly disagree; $7=$ Strongly agree) that allows calculating four scores: OVERALL (the overall satisfaction score), SYSUSE (system usefulness), INFOQUAL (information quality), and INTERQUAL (interface quality) [7]. All score results are higher for MobIES: OVERALL (MobIES: 6.37; MO: 5.08), SYSUSE 
(MobIES: 6.37; MO: 5.25), INFOQUAL (MobIES: 6.30; MO: 5.18), and INTERQUAL (MobIES: 6.34; MO: 4.58). The statements with the largest differences in the ratings cover the issues of system interface and task efficiency (see Fig. 6). S1 and S2 both indicate that participants appreciated the extended interface spanning across two screens as it was perceived as significantly more pleasant to use $(\mathrm{z}=-3.2$, $\mathrm{p}=.001)$ and the organization of information was rated to be more clear $(\mathrm{z}=-2.6$, $\mathrm{p}=.01)$. S3, S4, and S5 show that participants perceived MobIES as significantly more effective $(\mathrm{z}=-2.7, \mathrm{p}=.007)$, efficient $(\mathrm{z}=-2.4, \mathrm{p}=.01)$, and faster to use $(\mathrm{z}=-2.6, \mathrm{p}=.008)$.

(S1) The interface of this system was pleasant.

(S2) The organization of information on the system screens was clear.

(S3) I was able to efficiently complete the tasks and scenarios using this system.

(S4) I was able to complete the tasks and scenarios quickly using this system.

(S5) I could effectively complete the tasks and scenarios using this system.

Mobile \& Screen

- Mobile Only

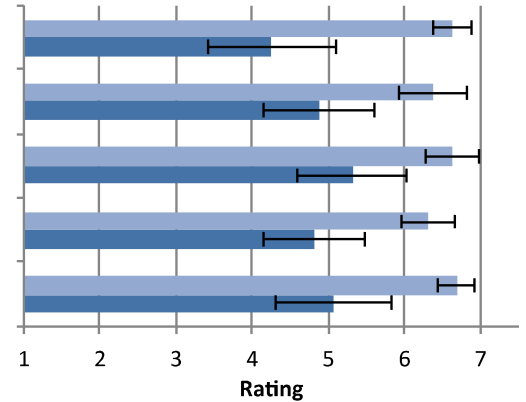

(1=Strongly disagree; $7=$ =Strongly agree)

Fig. 6. Questionnaire statements with the largest differences in ratings

Six of the participants emphasized that they liked the level of clarity achieved through the larger screen space. Also, participants pointed out that extending the interface of mobile applications would be helpful to show or share information with others. One user suggested a holder for the mobile phone to leave both hands available for interaction. Four participants pointed out that they liked the ease of use of the system. For instance, P8 stated "It is very easy to switch between using only the mobile phone and using the additional display." Few participants pointed out that they initially had to look for information after the user interface spanned across two displays. Yet all participants learned how to use the system quickly after a short introduction. Other participants highlighted that they liked the extension but expressed doubts whether an external display would be available when needed.

\section{$7 \quad$ Discussion and Conclusion}

MobIES addresses the issue that mobile users temporarily have the need for more screen space in selected situations, for instance, to gain more clarity when viewing large images or maps. The results of our laboratory study strongly indicate that users benefit from using this approach. Parameters that could not be mapped through an experimental setting, such as availability of matching external screens, as well as possible privacy and security concerns need to be considered when deploying such a system. The presented approach is based on a novel application of NFC technology that allows extending existing displays at very low costs. It enables users to take advantage of displays in their environments in order to extend the user interfaces of their mobile applications when needed. However, the presented implementation requires 
specific software to be available on the mobile and the stationary device, which limits the flexibility of users. To address this, future implementations could include a runtime environment on the stationary display system that executes application logic provided by the mobile client.

In a user study, we compared MobIES with the standard mobile phone option. The results indicate that participants appreciated the degree of information clarity, perceived their task performance to be faster, and highlighted that the system is easy to use. Future investigations will focus on providing a more generalized environment which allows users to take advantage of external displays that are not preconfigured.

Acknowledgement. This work has been conducted in the Emmy Noether research group Mobile Interaction with Pervasive User Interfaces funded by the German Research Foundation.

\section{References}

1. Baur, D., Boring, S., Feiner, S.: Virtual projection: exploring optical projection as a metaphor for multi-device interaction. In: CHI 2012. ACM (2012)

2. Chittaro, L.: Visualizing information on mobile devices. Computer 39(3), 40-45 (2006)

3. Greenberg, S., Boyle, M., Laberge, J.: PDAs and shared public displays: Making personal information public, and public information personal. PUC 3, 54-64 (1999)

4. Grolaux, D., Vanderdonckt, J., Van Roy, P.: Attach me, detach me, assemble me like you work. In: Costabile, M.F., Paternó, F. (eds.) INTERACT 2005. LNCS, vol. 3585, pp. 198-212. Springer, Heidelberg (2005)

5. Hardy, R., Rukzio, E.: Touch \& Interact: Touch-based interaction of mobile phones with displays. In: Proc. MobileHCI, pp. 245-254 (2008)

6. Hinckley, K., Ramos, G., Guimbretiere, F., Baudisch, P., Smith, M.: Stitching: Pen Gestures That Span Multiple Displays. In: AVI 2004, pp. 23-31. ACM (2004)

7. Lewis, J.R.: IBM computer usability satisfaction questionnaires: psychometric evaluation and instructions for use. Int. J. Hum.-Comp. Interact. 7(1), 57-78 (1995)

8. Lucero, A., Holopainen, J., Jokela, T.: Pass-Them-Around: Collaborative Use of Mobile Phones for Photo Sharing. In: CHI 2011, pp. 1787-1796. ACM (2011)

9. Lyons, K., Pering, T., Rosario, B., Sud, S., Want, R.: Multi-Display Composition: Supporting Display Sharing for Collocated Mobile Devices. In: Gross, T., Gulliksen, J., Kotzé, P., Oestreicher, L., Palanque, P., Prates, R.O., Winckler, M. (eds.) INTERACT 2009. LNCS, vol. 5726, pp. 758-771. Springer, Heidelberg (2009)

10. Rashid, U., Nacenta, M.A., Quigley, A.: The cost of display switching: a comparison of mobile, large display and hybrid ui configurations. In: AVI 2012. ACM (2012)

11. Reilly, D., Rodgers, M., Argue, R., Nunes, M., Inkpen, K.: Marked-up maps: combining paper maps and electronic information resources. PUC 10(4), 215-226 (2006)

12. Rekimoto, J., Saitoh, M.: Augmented Surfaces: A Spatially Continuous Work Space for Hybrid Computing Environments. In: CHI 1999, pp. 378-385. ACM (1999)

13. Schmidt, D., Seifert, J., Rukzio, E., Gellersen, H.: A cross-device interaction style for mobiles and surfaces. In: DIS 2012, pp. 318-327. ACM (2012)

14. Schneider, D., Seifert, J., Rukzio, E.: Mobies: extending mobile interfaces using external screens. In: Extended Abracts of MUM 2012, pp. 59:1-59:2. ACM (2012) (Demo Paper)

15. Ullmer, B., Ishii, H., Glas, D.: mediablocks: physical containers, transports, and controls for online media. In: SIGGRAPH 1998, pp. 379-386. ACM (1998) 